\title{
Excited states of ring-shaped (InGa)As quantum dots in a GaAs/(AIGa)As quantum well
}

\author{
F. Pulizzi,* D. Walker, A. Patanè, L. Eaves, and M. Henini \\ School of Physics and Astronomy, University of Nottingham, Nottingham NG7 2RD, United Kingdom \\ D. Granados and J. M. Garcia \\ Instituto de Microelectrònica de Madrid, CNM, CSIC, Isaac Newton, 8, Tres Cantos, 28760 Madrid, Spain \\ V. V. Rudenkov, P. C. M. Christianen, and J. C. Maan \\ High Field Magnet Laboratory, Institute for Molecules and Materials, Radboud University Nijmegen, Toernooiveld 7 , \\ 6525 ED Nijmegen, The Netherlands \\ P. Offermans and P. M. Koenraad \\ Department of Semiconductor Physics, Eindhoven University of Technology, P.O. Box 513, NL-5600 MB Eindhoven, The Netherlands \\ G. Hill \\ Department of Electronic and Electrical Engineering, University of Sheffield, Sheffield S3 3JD, United Kingdom
}

(Received 11 March 2005; published 2 August 2005)

\begin{abstract}
By studying the resonant tunneling of electrons through self-assembled ring-shaped (InGa)As quantum dots embedded in the quantum well of an $(\mathrm{AlGa}) \mathrm{As} / \mathrm{GaAs} /(\mathrm{AlGa}) \mathrm{As}$ heterostructure, we find evidence for the existence of excited states of the quantum dots which exhibit a $2 p_{z}$-like character along the growth direction $z$. The states are investigated by magnetotunneling spectroscopy with magnetic fields applied parallel and perpendicular to $z$.
\end{abstract}

DOI: 10.1103/PhysRevB.72.085309

PACS number(s): 73.23.-b, 73.63.Kv, 73.40.Gk

In self-assembled semiconductor quantum dots (QDs), carriers are confined in all three spatial dimensions. This leads to a spectrum of discrete energy levels, similar to that of atoms. ${ }^{1-7}$ The analogy with atoms is strengthened by the observation of excitonic and electronic states with $s-, p-$, and $d$-like character, all of which have been observed in optical ${ }^{6}$ and tunneling 8,9 spectroscopy studies. In addition, optical pumping experiments have provided evidence for shell-like filling of the electronic orbitals. ${ }^{6}$ However, in contrast to a real atom, a QD has a highly anisotropic confining potential, since its dimensions in the plane of growth (the wetting layer plane $x-y$ ) are typically an order of magnitude larger than that along the growth direction $(z)$. Hence spectroscopic investigations have been restricted mainly to states for which the excitations are confined to the $x-y$ plane.

In this paper, we study the electronic properties of QDs with an unusual ring-shape morphology, which can be generated under special growth conditions. We report experimental evidence for the presence of excited QD states with a $2 p_{z}$-like character. States with $2 p_{z}$ character have been observed previously for $\mathrm{Si}$ donors in a GaAs QW. ${ }^{10}$ For this study we use resonant tunneling heterostructure diodes in which a single layer of self-assembled ( $\mathrm{InGa})$ As quantum dots is embedded in an (AlGa)As/GaAs/(AlGa)As quantum well. The $2 p_{z}$-like quasibound states are revealed by the presence of additional resonant peaks in the tunnel current. By combining photoluminescence and magnetotunneling spectroscopy with magnetic field $B$ applied along $z$ and in the $x-y$ plane, we show that these resonances correspond to electron tunneling into $2 p_{z}$-like states with binding energies in the range 5-30 meV below the edge of the continuum of the first excited two-dimensional (2D) subband of the GaAs QW.
Despite the ring-shape structure of the dots, we find no evidence in our tunneling data for states showing AharonovBohm character.

Our device is a GaAs $/ \mathrm{Al}_{0.4} \mathrm{Ga}_{0.6}$ As double barrier electron resonant tunneling diode (RTD) grown by molecular beam epitaxy (MBE) on a (100)-oriented $n^{+}$GaAs substrate. The conduction band profile of the device and the QD energy levels are shown schematically in Fig. 1(a). Two 8-nm-thick $\mathrm{Al}_{0.4} \mathrm{Ga}_{0.6}$ As tunnel barriers define a $10-\mathrm{nm}$-thick GaAs QW, which contains a layer of ( $\mathrm{InGa})$ As QDs. Undoped GaAs spacer layers of width $50 \mathrm{~nm}$ separate the $\mathrm{Al}_{0.4} \mathrm{Ga}_{0.6} \mathrm{As}$ barriers from lightly $n$-doped $\left(n=2 \times 10^{17} \mathrm{~cm}^{-3}\right)$ GaAs layers of width $50 \mathrm{~nm}$. Two $0.4 \mu \mathrm{m} n^{+}$Si-doped GaAs layers form the outer electrical contacts of the device. The QW and QD layers were prepared as follows: after the growth of the first half of the QW, 1.85 monolayers of InAs were deposited to form the QD layer. The dots were then capped with $2 \mathrm{~nm}$ of GaAs and annealed for $90 \mathrm{~s}$ in an $\mathrm{As}_{2}$ atmosphere. The GaAs growth was then resumed and the remaining $3 \mathrm{~nm}$ of the well was grown. This procedure leads to the transformation of the InAs layer into (InGa)As ring-shaped dot structures, which are commonly referred to as quantum rings (QRs). ${ }^{11}$ For comparison, experiments were also carried out on a control sample (C sample), grown with the same structure as the QD sample but without the InAs layer. The structures were processed into mesas with diameters between 50 and $200 \mu \mathrm{m}$, with an anular metallic top contact layer to provide optical access.

Atomic force microscope images of similarly prepared uncapped ring-shaped QD layers (with the growth terminated after the annealing stage) reveal a QD density of 7 $\times 10^{9} \mathrm{~cm}^{-2}$, see inset of Fig. 1(a). Cross-sectional scanning 
(a)

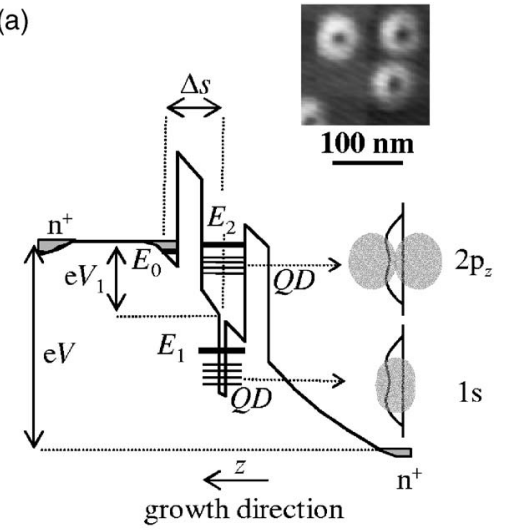

(b)

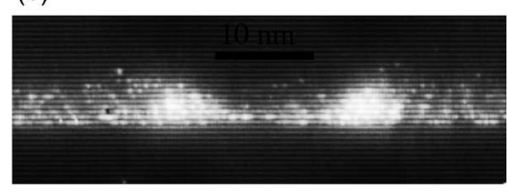

FIG. 1. (a) Schematic conduction-band profile under bias of our RTD incorporating ring-shaped (InGa)As QDs. The right inset indicates the wave-function symmetry of the $1 s$ (bottom) and $2 p_{z}$ (top) quasibound states of the QDs. Upper inset: AFM image of uncapped ring-shaped QDs. (b) XSTM image $(50 \times 17 \mathrm{~nm})$ of a capped ring-shaped QD. The light areas are In-rich. The atomic layers are fully resolved.

tunneling microscope (XSTM) images of similarly GaAscapped ring-shaped QDs indicate a lateral diameter of $\approx 20 \mathrm{~nm}$ and a height of $3 \mathrm{~nm}$ [Fig. 1(b)]. ${ }^{12}$ Ring-shaped QDs have shown a very high intraband oscillator strength ${ }^{13}$ and a large excitonic electric dipole. ${ }^{14}$ Capacitance-voltage experiments in magnetic fields have also provided some evidence of Aharonov-Bohm states, related to magnetic flux quantization in the ring-shaped structure. ${ }^{15}$

Figure 2 compares the current-voltage characteristics, $I(V)$, of the QD and $\mathrm{C}$ samples, measured on $50 \mu \mathrm{m}$ mesas. The $\mathrm{C}$ sample exhibits two strong resonant peaks in both

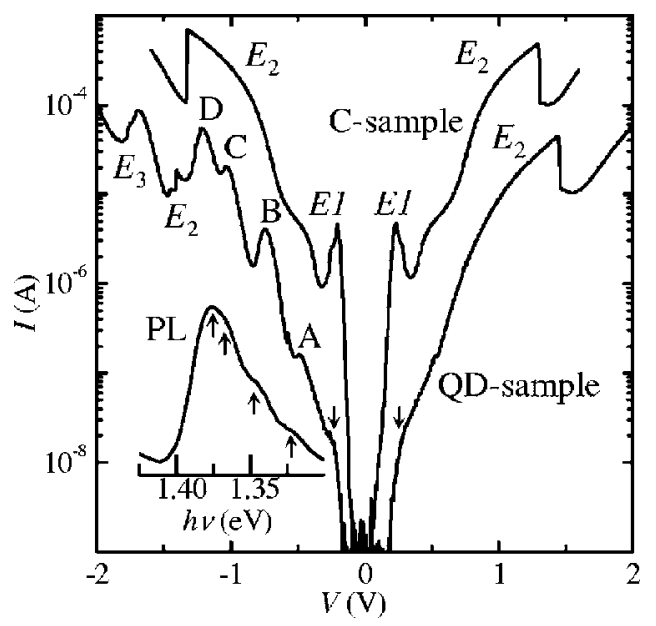

FIG. 2. $I(V)$ characteristics at $T=4.2 \mathrm{~K}$ of the QD and control (C) samples. The inset shows the photoluminescence spectrum at $T=10 \mathrm{~K}$ measured at zero bias for the QD sample. forward and reverse bias, corresponding to electron tunneling into the $E_{1}$ and $E_{2}$ two-dimensional (2D) subbands of the QW [Fig. 1(a)]. Some of the features of the QD sample are typical of RTDs incorporating QDs. For example, the current in the QD sample is approximately one order of magnitude lower than in the $\mathrm{C}$ sample. ${ }^{9}$ This is attributed to the fact that most of the electrons that tunnel into the QW relax down to the lower energy states of the QDs. They then tunnel into the collector through the second barrier which has a relatively large height and low transmission coefficient. Secondly, the resonant peak at $\pm 0.1 \mathrm{~V}$ observed in the $I(V)$ curve of the $\mathrm{C}$ sample, which arises from resonant tunneling of electrons into the lowest QW subband, $E_{1}$, appears as a much weaker shoulder at $\pm 0.1 \mathrm{~V}$ (indicated by the downward arrows in Fig. 2) in the $I(V)$ of the QD sample. We attribute this shoulder to resonant tunneling into the lowest energy 2D subband of the QW which is formed by the combination of the residual (InGa)As wetting layer (WL) potential and the $\mathrm{GaAs} / \mathrm{Al}_{0.4} \mathrm{Ga}_{0.6} \mathrm{As} \mathrm{QW}$ potential. In the QD sample, the disorder associated with the QD layer disrupts the low $k$ states of the WL subband and tends to broaden and suppress the associated resonance in $I(V) .{ }^{16}$ Evidence for this attribution is provided by the magnetic field dependence of the $I(V)$ curves as described later in the paper.

In forward bias, the QD sample shows a strong resonance associated with tunneling into the second QW subband $E_{2}$. The corresponding peak in reverse bias is much weaker. A resonant peak for bias $\approx-1.7 \mathrm{~V}$ is also observed in the $I(V)$ of the QD sample. We attribute this to resonant tunneling of electrons into the highest QW subband, $E_{3}$. In the $\mathrm{C}$ sample, the $E_{3}$ subband is expected to be too high in energy to be revealed in $I(V)$. In contrast, the introduction of the InAs layer lowers the energy of the $E_{3}$ subband below the top of the barriers, giving rise to a resonant peak in $I(V)$.

We have examined the low bias-low current $(1-100 \mathrm{pA})$ part of the $I(V)$ characteristics in some detail but found no evidence of peaks corresponding to resonant tunneling into the ground states of the QDs, which have been reported previously in other resonant tunneling devices incorporating QDs. ${ }^{8,9,17,18}$ We attribute this to the fact that the energy levels of the QD ground states lie below the chemical potential in the emitter at all biases so energy-conserving tunneling transitions through the dots are not allowed.

We now focus on the most striking feature of the $I(V)$ characteristics of the QD sample, namely the presence of four additional resonant peaks in reverse bias, in the range between the $E_{1}$ and the $E_{2}$ subband resonant peaks. We have measured the $I(V)$ curves of 30 different mesas with different diameters, taken from different sections of the $5 \mathrm{~cm}$ diameter MBE wafer. We observe this series of additional resonant peaks in all 30 mesas. Moreover, all of the mesas have essentially identical current-density characteristics, $J(V)$, when the measured current is divided by the cross-sectional area of the mesas. This result indicates that the peaks are due to a macroscopic property of the MBE wafer and not to local or mesoscopic effects of a given mesa.

The inset of Fig. 2 shows the quantum dot photoluminescence (PL) spectrum obtained at zero bias, with excitation by the $632.8 \mathrm{~nm}$ line of a HeNe laser. The PL spectrum consists 
of four main components indicated by upward arrows in the inset. The overall spectral shape is independent of the excitation power $\left(0.1-100 \mathrm{~W} \mathrm{~cm}^{-2}\right)$, which suggests that these bands do not arise from higher energy levels due to state filling or electron charging effects. We therefore attribute the PL features to ground state-to-ground state interband transitions from a multimodal distribution of QDs consisting of four different sizes and/or composition. Evidence of a similar multimodal distribution of QDs has been reported recently. ${ }^{19}$

It can be seen that the energy spacing between the peaks in PL is roughly proportional to the energy spacing of peaks $A$ to $D$ in the $I(V)$ curves. A one-to-one correspondence can be made between the PL components and peaks $A$ to $D$, and it suggests that each of the peaks in $I(V)$ is associated with one of the four sets of dot distributions indicated by the PL spectrum.

In order to study the character of peaks $A$ to $D$ we use magnetotunneling spectroscopy (MTS). In MTS, a magnetic field is applied parallel to the QW plane and is used to tune the in-plane momentum $\hbar k$ of the tunneling carrier (electron or hole); the energy of the tunneling electron is adjusted by the applied bias. By studying the dependence of the voltage position of the resonant peaks in $I(V)$ as a function of inplane magnetic field, $B_{x y}$, the energy-wave-vector dispersion curve, $\epsilon(k)$, of the QW can be mapped. ${ }^{20}$ Also for tunneling into localized states in the QW, the dependence of the amplitude of the resonant peaks on $B_{x y}$ provides a powerful means for imaging the electron wave function. ${ }^{8,9}$

Figure 3(a) shows the effect on the $I(V)$ curves of $B_{x y}$. With increasing $B_{x y}$, the weak shoulder associated with electron tunneling into the wetting layer subband $E_{1}$ develops into a well-defined resonant peak, as has been reported in earlier experiments on RTDs incorporating QDs. ${ }^{16}$ The magnetic field $B_{x y}$ provides the tunneling electrons with an inplane momentum $\hbar k=e B_{x y} \Delta s$, where $\Delta s$ is the tunneling distance [see Fig. 1(a)]. The additional kinetic energy associated with this momentum is provided by the applied bias. At sufficiently large values of $k\left(\geqslant 10^{8} \mathrm{~m}^{-1}\right)$ the QD-induced disorder has less effect on the $E_{1}$ subband states and the resonant tunneling peak in $I(V)$ is restored. Also note that all of the resonances shift to higher negative bias with increasing $B_{x y}$. However, while the amplitude of the $E_{1}$ resonance increases as expected for tunneling into a QW subband, ${ }^{16,20}$ that of peaks $A$ to $D$ falls off with increasing $B_{x y}$ and, at $11 \mathrm{~T}$, the peaks are barely visible in $I(V)$.

The $I(V)$ curves measured in magnetic field $B_{z}$, applied parallel to the growth direction [see Fig. 3(b)] also reveal the difference in character of peaks $A$ to $D$ compared to peak $E_{1}$. Peaks $A$ to $D$ are only slightly affected by $B_{z}$, whereas the $E_{1}$ resonance splits due to the formation of Landau levels (LLs) in the QW. An extra peak due to resonant tunneling of electrons from the emitter into the second $(N=1)$ LL appears for $B_{z} \geqslant 18 \mathrm{~T}$, in addition to the principal resonance associated with $N=0$ [arrows in Fig. 3(b)].

The absence of LL splitting for peaks $A$ to $D$ with increasing $B_{z}$ indicates that they are due to electron tunneling into localized states rather than into bandlike states. This is supported by the observation of a decrease in amplitude of these peaks with increasing $B_{x y}$, which has been observed and in-
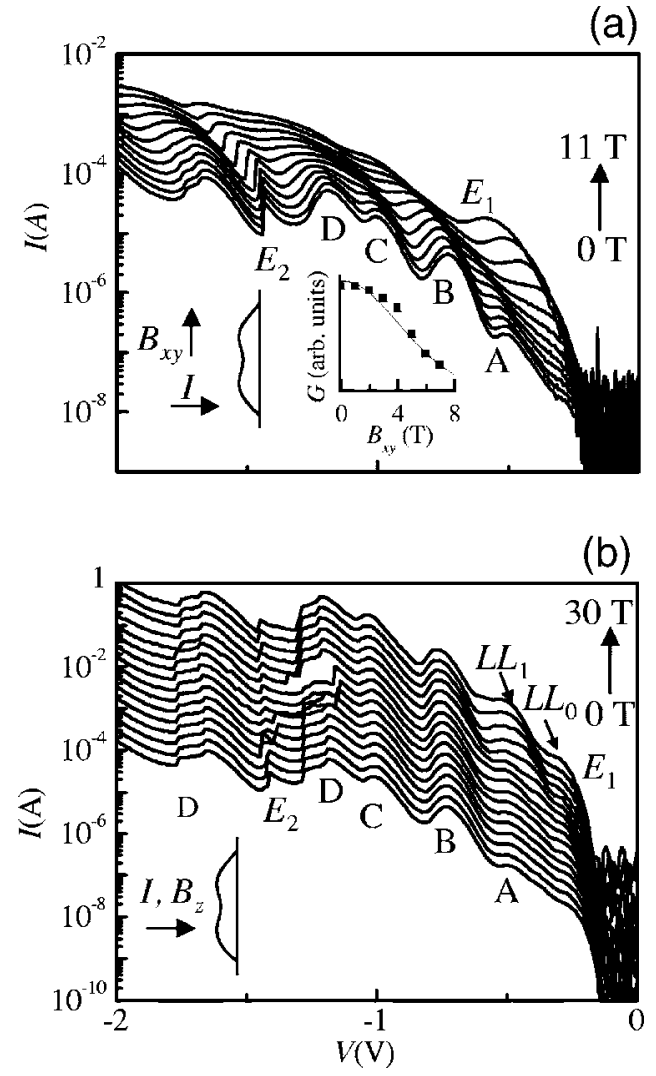

FIG. 3. $I(V)$ curves for QD sample at different magnetic fields. (a) The field $B_{x y}$ is applied parallel to the QW plane. $B_{x y}$ is increased from 0 (bottom curve) to $11 \mathrm{~T}$ (top curve) in $1 \mathrm{~T}$ steps. The inset shows the dependence of the amplitude of the differential conductance $(G=d I / d V)$ on $B_{x y}$ for peak $A$. (b) The field $B_{z}$ is perpendicular to the $\mathrm{QW}$ plane. $B_{z}$ is increased from 0 (bottom curve) to $30 \mathrm{~T}$ (top curve) in $2 \mathrm{~T}$ steps. In both panels the curves are displaced along the vertical axis for clarity.

vestigated in detail for electron tunneling into conventional self-assembled quantum dots ${ }^{9}$ and impurity states. ${ }^{21}$ The inset of Fig. 3(a) shows the dependence of the amplitude of the differential conductance $(G=d I / d V)$ on $B_{x y}$ for peak $A$. Peaks $B, C$, and $D$ show similar dependence. The monotonic Gaussian-like decrease of the amplitude with increasing $B_{x y}$ effectively maps out the square of the amplitude $|\psi(k)|^{2}$ of the eigenfunctions in $k$-space (where $k$ is in the quantum well plane). This indicates that the wave functions of these states have no nodes along $x-y$. At $B_{x y} \approx 5 \mathrm{~T}$, the amplitude of each peak decreases to one half of its zero field value. This indicates that the wave function has a typical spatial extent (full width at half maximum) along $x-y$ of $10 \mathrm{~nm} .{ }^{9}$ Consistent with the Gaussian shape of the wave function along $x-y$, the lack of any peak splitting with increasing $B_{z}$ indicates the absence of an orbital Zeeman effect and that the localized states have orbital angular momentum component $\left\langle\ell_{z}\right\rangle=0$.

To determine the energy, magnetic field dependence, and character of the states associated with peaks $A$ to $D$, we need to consider the way in which the applied voltage is distributed across the device. We define an electrostatic leverage factor, $f$, as the ratio between the total applied voltage $V$ and the portion $V_{1}$ dropped between the emitter and the center of 

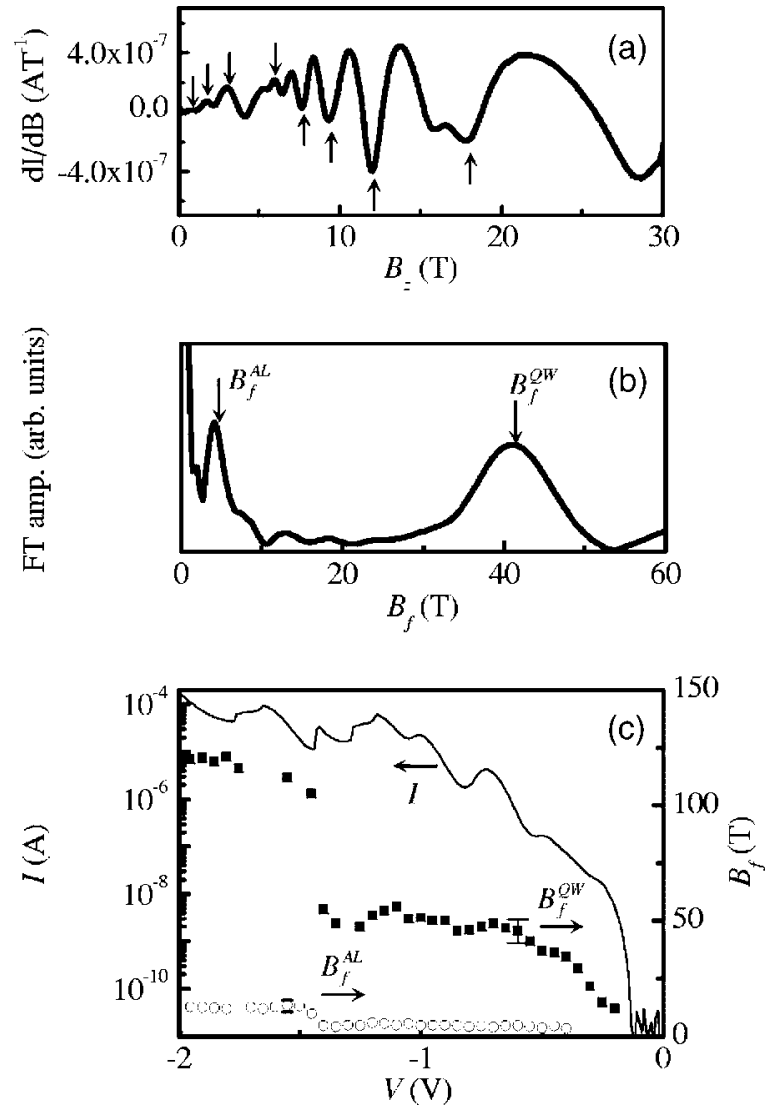

FIG. 4. (a) $\partial I / \partial B_{z}$ curve measured for $V=-0.6 \mathrm{~V}$ in the QD sample. Two series of magneto-oscillations are observed, indicated by upward and downward arrows. (b) Fourier spectrum of the magneto-oscillations in (a) plotted as a function of the fundamental field $B_{f}$, as described in the text. The two series of magnetooscillations revealed by the Fourier spectrum have fundamental fields, $B_{f}^{Q W}$ and $B_{f}^{A L}$. (c) Voltage dependence of $B_{f}^{Q W}$ and $B_{f}^{A L}$. The $I(V)$ curve at $B_{z}=0$ is shown for comparison.

the QW, $f=V / V_{1}$. This ratio is determined partly by the geometry of the device, but is also bias-dependent as it is influenced by the charge accumulated in the QW. ${ }^{22}$ For example, when electrons start tunneling out of the emitter, electron charge builds up in the QW and effectively pins the Fermi level in the emitter to the resonant state in the QW, so that most of the additional applied bias is dropped between the well and the collector. This pinning persists up to the bias point at which the QW discharges and the device switches to an off-resonant state.

The effect on $f$ of the charge build-up in the QW can be determined from experiment by analyzing the magnetooscillations in the current as a function of $B_{z}$ for fixed applied bias. ${ }^{22}$ Figure 4(a) shows a typical plot of the differential magnetocurrent $\partial I / \partial B_{z}$ vs $B_{z}$, measured for an applied bias of $-0.6 \mathrm{~V}$. The curve exhibits two series of magnetooscillations, each with well-defined period $\Delta\left(B_{z}^{-1}\right)$. Figure 4(b) shows the Fourier spectrum of these magnetooscillations, revealing two clear peaks. We define a fundamental magnetic field $B_{f}$ for each series given by $B_{f}^{-1}$ $=\Delta\left(B_{z}^{-1}\right)$.

The series of magneto-oscillations at low magnetic fields with a small $B_{f}=B_{f}^{A L}$, indicated by downward arrows in Fig. $4(\mathrm{a})$, arises from sequential depopulation with increasing $B_{z}$ of the LLs of the degenerate two-dimensional electron gas in the emitter accumulation layer. For a fixed applied bias, the $B_{f}^{A L}$ value gives us the sheet density $n_{s}$ of the electron in the emitter accumulation layer according to the relation

$$
n_{s}(V)=2 e B_{f}^{A L}(V) / h \text {. }
$$

The magneto-oscillations at higher magnetic fields, indicated by upward arrows in Fig. 4(a), are due to electrons tunneling from the emitter accumulation layer $E_{0}$ into the LLs associated with the $E_{1}$ subband of the QW. At constant bias and with increasing $B_{z}$, a resonant feature appears in the current when the following energy conservation condition is satisfied:

$$
E_{0}-E_{1}=N \hbar e B_{z} / m_{\mathrm{c}}+\left(\hbar \omega_{\mathrm{LO}}\right) .
$$

Here $m_{\mathrm{c}}$ is the in-plane (or cyclotron) mass in the QW, $N$ is an integer, and the term in brackets indicates the possibility of the emission of a longitudinal optical (LO) phonon in the tunneling transition. This series is also periodic in $1 / B$ with a fundamental field

$$
B_{f}^{Q W}(V)=\left(E_{0}-E_{1}\right) m_{\mathrm{c}} / \hbar e,
$$

for the case when no LO phonons are emitted. Since $\left(E_{0}\right.$ $\left.-E_{1}\right) / e$ is the voltage drop across the effective width $\Delta s$ of the first tunnel barrier, the electrostatic leverage of the device is simply $f=m_{\mathrm{c}} V / B_{f}^{Q W}(V)$. It is also interesting to note that since $\left(E_{0}-E_{1}\right)$ depends linearly on $n_{s}$, the values of $B_{f}^{Q W}$ and $B_{f}^{A L}$ obey the simple relation

$$
\partial B_{f}^{Q W} / \partial B_{f}^{A L}=e^{2} m_{\mathrm{c}} \Delta s / \pi \epsilon \epsilon_{0} \hbar^{2}=4 \Delta s / a_{0},
$$

where $a_{0}=10 \mathrm{~nm}$ is the Bohr radius in GaAs. A simple electrostatic model based on the Fang-Howard variational wave function gives the effective tunnel distance from the emitter into the QW, $\Delta s=26 \mathrm{~nm}$ at $V=-1 \mathrm{~V}$, so that $\partial B_{f}^{Q W} / \partial B_{f}^{A L}$ $\simeq 10$. This relation agrees with the experimental value over the full bias range.

At low bias $(\leqslant 0.5 \mathrm{~V})$, the measured value of $B_{f}^{Q W}$ gives $f \approx 4$, which agrees with that determined by a simple electrostatic model and the layer composition of our device. For higher biases, corresponding to resonances $A$ to $D, B_{f}^{Q W}$ increases more slowly with $V$, thus indicating charge build-up in the QW and a larger value of $f(f \approx 10$ at $V=-1 \mathrm{~V})$. The large jump in the measured value of $B_{f}^{Q W}$ and $B_{f}^{A L}$ at a voltage of $\approx-1.4 \mathrm{~V}$, where the current falls abruptly just beyond the resonant peak $E_{2}$, indicates the discharge of electrons from the QW and the corresponding increase in $n_{s}$, which is required to maintain the voltage drop across the device.

This analysis allows us to convert the voltage position of different resonances in $I(V)$ into an energy scale for the electron states in the QW. Figure 5(a) shows the magnetic field dependence of the energy of the quantum states associated with the resonant features in $I(V)$ for $B$ perpendicular to $z$. Two features of these plots are noteworthy. First, the parabolic shift of $E_{1}$ to higher energies with increasing $B_{x y}$, i.e., increasing $k$, indicates that this feature corresponds to tunneling into energy-dispersed $k$ states of the QW subband [see 

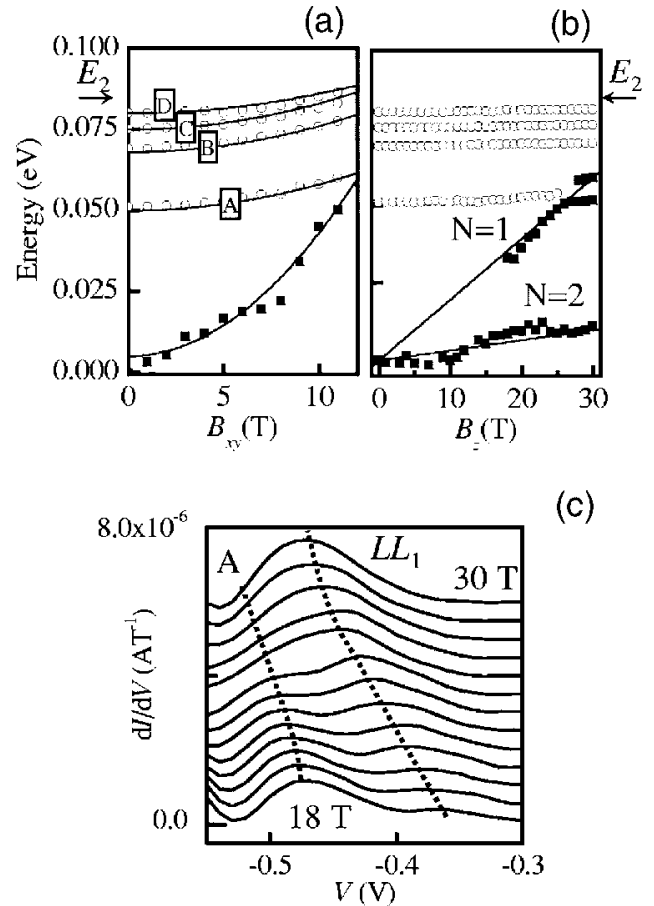

(c)

FIG. 5. (a) and (b) Dependence of the energies of the electron states in the QW on (a) $B_{x y}$ and (b) $B_{z}$ as measured relative to the edge of the $E_{1}$ subband. (c) Detail of the differential conductance of the QD sample showing the anticrossing between the $N=1$ Landau level peak and peak $A$.

continuous line in Fig. 5(a)]. The magnitude of the energy shift $\Delta \epsilon$ is described by the relation $\Delta \epsilon=\hbar^{2} k^{2} / 2 m_{\mathrm{c}}$, where $k=e B_{x y} \Delta s / \hbar$ and $\Delta s \approx 26 \mathrm{~nm}$ is the effective tunneling distance. Second, peaks $A$ to $D$ have a much smaller shift with $B_{x y}$, which is consistent with the small increase in diamagnetic energy of bound states confined in the QD potential along the $z$ direction. The diamagnetic shift in energy is $e B^{2}$ $\left\langle\Delta z^{2}\right\rangle / 2 m_{\mathrm{QD}}$, where $\Delta z=z-z_{0}, z_{0}$ is the mean position of the bound electron along $z$, and $m_{\mathrm{QD}}$ is the effective mass for the dot. By assuming a value of $m_{\mathrm{QD}}$ ranging between that for bulk GaAs and InAs, we obtain a value of $\left\langle\Delta z^{2}\right\rangle^{1 / 2}$ between 4 and $7 \mathrm{~nm}$. This is larger than the height of the QD along $z$ $(3 \mathrm{~nm})$ and indicates that the electron wave function for these states extends well beyond the physical boundaries of the dot. We do not observe any indication of in-plane anisotropy, since changing the in-plane orientation of $B_{x y}$ does not affect the shape of the $I(V)$ curve.

Additional useful information can be obtained from the data shown in Fig. 5(b), where the energy positions of the resonant states are plotted against $B_{z}$. This orientation of the magnetic field gives rise to LL splitting and should lift any orbital angular momentum degeneracy of bound states with an energy splitting $\hbar \omega_{\mathrm{c}} \cong 16.5 \mathrm{meV}$ at $B_{z}=10 \mathrm{~T}$. We have already alluded to the LL splitting of the $E_{1}$ subband. The magnitude of the LL splitting gives an effective in-plane cyclotron mass $m_{\mathrm{c}} \cong 0.07 m_{0}$. This value agrees with that expected for bulk GaAs and provides additional support of our analysis of the electrostatic leverage factor. Furthermore, we note that the upper $N=1 \mathrm{LL}$ appears to show a weak anticrossing with the QD state associated with the resonance $A$.

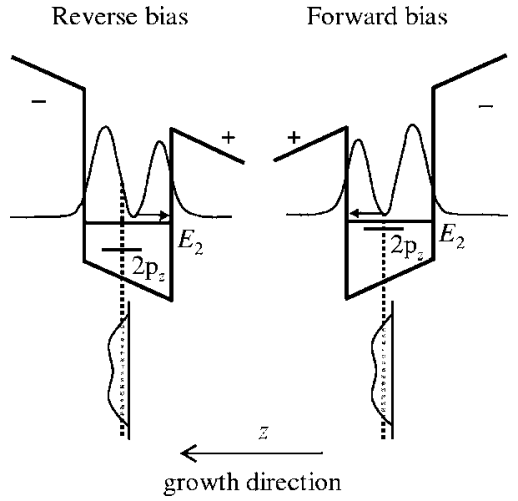

FIG. 6. Diagram showing the possible effect of reverse and forward bias on the $E_{2}$ subband. The probability density is obtained from an effective mass calculation of the energy eigenstates of a 10-nm-thick GaAs/ $\mathrm{Al}_{0.4} \mathrm{Ga}_{0.6} \mathrm{As} \mathrm{QW}$ in the presence of a forward and reverse electric field corresponding to that of our experiment.

The crossover of amplitudes of these two states with increasing $B_{z}$, which is characteristic of quantum interaction, is evident from Fig. 5(c). The magnitude of the anticrossing energy suggests that the coupling of the cyclotron motion with the $z$-motion is relatively small $\left(\ll \hbar \omega_{\mathrm{c}}\right.$ at the anticrossing point).

Based on this analysis of the data we can now build up a model for the electronic states in the QW. The four additional resonances $A$ to $D$ in $I(V)$ and the four component-PL spectra indicate a multimodal distribution of QD sizes, distributed into four main size groups. We propose that peaks $A$ to $D$ arise from resonant tunneling of electrons into discrete excited state energy levels associated with the QDs. These states are well above the edge of the $E 1$ subband but are quasibound with respect to the edge of the $E_{2}$ QW subband with a small binding energy $(\leqslant 30 \mathrm{meV})$. Their eigenfunctions have $2 p_{z}$-like $\left(\ell_{z}=0\right)$ character, and would therefore have two lobes along $z$, as indicated schematically in Fig. 1(a). The corresponding electronic $1 s$ ground states of the QDs are bound at larger energies $(\approx 100 \mathrm{meV})$ with respect to the edge of $E_{1}$.

A puzzling aspect of the $I(V)$ curves is the asymmetry with respect to bias and the complete absence of the resonant peaks in forward bias corresponding to $A, B, C$, and $D$. Note that the $E_{2}$ resonance in forward bias in the QD sample is as well-defined as in the control sample, indicating that the InAs QD layer produces a negligible effect on the $E_{2}$ states at this bias. We suggest that, in this case, the node of the eigenfunction is very close to the InAs layer so that the perturbation on the electron tunneling into $E_{2}$ is minimal (see Fig. 6). In contrast, a reverse bias acts to shift the wave function away from the InAs layer. In this case, the probability density of the wave functions at the InAs layer could be sufficient to lead to weak binding of the $2 p_{z}$-like states to the QDs (see Fig. 6). The perturbing effect of the InAs layer could also explain the observed significant reduction of the amplitude of the $E_{2}$ resonance in reverse bias, as a sizeable fraction of the area of the heterostructure is influenced by the QD potential. This is evident from the AFM and XSTM images [Fig. 1(a)]. Therefore the number of pure $E_{2}$-subband 
$k$-states available for resonant tunneling is reduced.

The observation of the $2 p_{z}$-like states is not strictly related to the ring shape of the QDs and could be also revealed in conventional QDs, in principle. However, the QDs considered in this work appear to be distributed into four distinct and narrow energy ranges of a multimodal distribution, which we believe facilitates the observation of well-defined resonances in the current-voltage characteristics. In addition, the quantum rings extend significantly along the $z$ direction (their height is about $3 \mathrm{~nm}$ ), which should lead to a strong perturbation of the first excited state of the well and the formation of bound $2 p_{z}$-like states.

To summarize, we have investigated the electronic properties of ring-shaped self-assembled quantum dots using magnetotunneling and photoluminescence spectroscopy. We observe unusual resonant features in the current-voltage characteristics and provide evidence that they arise from electron tunneling into quasibound quantum dot states with $2 p_{z}$-like character. These states have $\left\langle\ell_{z}\right\rangle=0$. Despite the ringlike shape of the dots, there is no evidence of magnetic flux quantization effects in the magnetotunneling spectra. Resonant voltage-controlled filling of these excited states could provide a means of exciting infrared intraband transitions of QDs.

This work is supported by the Engineering and Physical Sciences Research Council (United Kingdom), the Spanish MCYT under NANOSELF Project TIC2002-04096-C03-03, the SANDiE Network of Excellence of the European Commission, Contract No. NMP4-CT-2004-500101, and the EU 6th Framework "Structuring the European Research Area, Research Infrastructures Action," Contract No. RII3-CT2004-506239.
*Present address: Department of Physics and Astronomy, University of Sheffield, Sheffield S3 7RH, United Kingdom. Electronic address: f.pulizzi@sheffield.ac.uk

${ }^{1}$ J. Y. Marzin, J. M. Gerard, A. Izrael, D. Barrier, and G. Bastard, Phys. Rev. Lett. 73, 716 (1994).

${ }^{2}$ R. J. Notzel, J. Temmyo, and T. Tamamura, Nature (London) 369, 131 (2000).

${ }^{3}$ D. Bimberg and M. Grundmann, Quantum Dot Heterostructures (Wiley, New York, 1999).

${ }^{4}$ L. Landin, M. S. Miller, M. E. Pistol, C. E. Pryor, and L. Samuelson, Science 280, 262 (1998).

${ }^{5}$ Y. Toda, O. Moriwaki, M. Nishioka, and Y. Arakawa, Phys. Rev. Lett. 82, 4114 (2000).

${ }^{6}$ M. Bayer, O. Stern, P. Hawrylak, S. Fafard, and A. Forchel, Nature (London) 405, 923 (2000).

${ }^{7}$ R. J. Warburton, C. Schäflein, D. Haft, F. Bickel, A. Lorke, K. Karrai, J. M. Garcia, W. Schoenfeld, and P. M. Petroff, Nature (London) 405, 926 (2000).

${ }^{8}$ E. E. Vdovin, A. Levin, A. Patanè, L. Eaves, P. C. Main, Y. N. Khanin, Y. V. Dubrovskii, M. Henini, and G. Hill, Science 290, 122 (2000).

${ }^{9}$ A. Patanè, R. J. A. Hill, L. Eaves, P. C. Main, M. Henini, M. L. Zambrano, A. Levin, N. Mori, C. Hamaguchi, Y. V. Dubrovskii, E. E. Vdovin, D. G. Austin, S. Tarucha, and G. Hill, Phys. Rev. B 65, 165308 (2002).

${ }^{10}$ M. Helm, F. M. Peeters, F. De Rosa, E. Colas, J. P. Harbison, and L. T. Florez, Phys. Rev. B 43, 13983 (1991).

${ }^{11}$ D. Granados and J. M. Garcia, Appl. Phys. Lett. 82, 1 (2003).

${ }^{12}$ P. Offermans, P. M. Koenraad, J. H. Wolter, D. Granados, J. M. Garcia, V. M. Fomin, V. N. Gladilin, and J. T. Devreese (unpub- lished). The XSTM technique used is described in detail in D. M. Bruls, J. W. A. M. Vugs, P. M. Koenraad, H. W. M. Salemink, and J. H. Wolter, Appl. Phys. Lett. 81, 1708 (2002).

${ }^{13}$ R. J. Petterson, A. Lorke, K. Karrai, J. P. Kotthaus, J. M. Garcia, and P. M. Petroff, Physica E (Amsterdam) 6, 510 (2000).

${ }^{14}$ R. J. Warburton, C. Schulhauser, D. Haft, C. Schäflein, K. Karrai, J. M. Garcia, W. Schoenfeld, and P. M. Petroff, Phys. Rev. B 65, 113303 (2002).

${ }^{15}$ A. Lorke, R. J. Luyken, A. O. Govorov, J. P. Kotthaus, J. M. Garcia, and P. M. Petroff, Phys. Rev. Lett. 84, 2223 (2000).

${ }^{16}$ F. Pulizzi, E. E. Vdovin, K. Takehana, Y. V. Dubrovskii, A. Patanè, L. Eaves, M. Henini, P. N. Brunkov, and G. Hill, Phys. Rev. B 68, 155315 (2003).

${ }^{17}$ M. Narihiro, G. Yusa, Y. Nakamura, T. Noda, and H. Sakaki, Appl. Phys. Lett. 70, 105 (1997).

${ }^{18}$ T. Suzuki, K. Nomoto, K. Taira, and I. Hase, Jpn. J. Appl. Phys., Part 1 36, 1917 (1997).

${ }^{19}$ R. Heitz, F. Guffarth, K. Pötschke, A. Schliwa, D. Bimberg, N. D. Zakharov, and P. Werner, Phys. Rev. B 71, 045325 (2005).

${ }^{20}$ R. K. Hayden, D. K. Maude, L. Eaves, E. C. Valadares, M. Henini, F. W. Sheard, O. H. Hughes, J. C. Portal, and L. Cury, Phys. Rev. Lett. 66, 1749 (1991).

${ }^{21}$ J.-W. Sakai, T. M. Fromhold, P. H. Beton, L. Eaves, M. Henini, P. C. Main, F. W. Sheard, and G. Hill, Phys. Rev. B 48, 5664 (1993).

${ }^{22}$ L. Eaves, G. A. Toombs, F. W. Sheard, C. A. Payling, M. L. Leadbeater, E. S. Alves, T. J. Foster, P. E. Simmonds, M. Henini, O. H. Hughes, J. C. Portal, G. Hill, and M. A. Pate, Appl. Phys. Lett. 52, 212 (1988). 